International Journal of Engineering \& Technology, $7(2.8)(2018) 612-616$
International Journal of Engineering \& Technology
SPC
Website: www.sciencepubco.com/index.php/IJET
Research Paper

\title{
IoT- save the community project
}

\author{
N. Deepa, Rakshit Agarwal, Rohan Uprety, Anubhab Biswas, A. Venkat Krishna \\ Electronics and Instrumentation, SRM University, Chennai,India \\ *Corresponding Author Email: deepa.n@ktr.srmuniv.ac.in
}

\begin{abstract}
IoT offers elaborate structures that combine multiple disperse additives towards their synergetic use. The machine suggested within this paper is an answer to track the environment conditions at a selected location while allowing the facts to be seen everywhere around the globe for the propagation of statistics in absence of any current infrastructure. Importance is given on how sensing and communique applications of IoT be utilized successfully in case of catastrophe control. The hardware of the module used for this motive is calculated and elaborated in a detailed way.
\end{abstract}

Keywords: IoT, RaspberryPi, Python, Disaster Management.

\section{Introduction}

Consistent with Federal Emergency Management Corporation (FEMA), an emergency is any catastrophic event like typhoon, twister, hurricane, flood, tidal wave, earthquake, volcanic eruption, tsunami, landslide, mudslide, snow fall, fireplace, explosion, nuclear accident, or any other natural or human-initiated catastrophe that drives motives to save lives and shield belongings, public health, and security in order to correctly manage the catastrophic occasions, intel needs to collaborate, for instance via sharing assets and/or facts and coordinating moves, decisions and activities.. The principal idea of IoT is the exchange of data among various devices. Attention has been lately grasped by the Internet-based sensor network. Servers all around the globe accumulate information which is obtained from various sensors like a motion sensor, temperature sensor, accelerometer, which are linked to the Cloud. Safety and manageability of data transmission and deploy ability of sensors connected to the net in a cordless manner are the predominant troubles though, low price and excessive scalability are expected. [2]. Our approach makes use of a Raspberry $\mathrm{Pi}$, for size and data acquisition of the encompassing environmental conditions which in turn facilitates in disaster management. on this prototype, we generally tend to construct a community in which every residence is equipped with Raspberry Pi linked with

diverse sensors which can be used to feel abnormalities in the surroundings. The response from the various sensors is then circulate-covered to the net of factors platform that's connected with a utility to visualize the data. SMS alert is sent to citizens of the house so one can discover time to searching for a safe haven. as an instance, every residence is attached with seismic sensors that are used to discover an earthquake and the message is then dispatched to the residents of the residence to alert them and to offer them time to react and searching for refuge. We've got additionally geared up the homes with warmness sensors to detect fireplace and we plan to sync them with the nearest fire stations for instant help. For citizens living in coastal regions, there is probably a possibility of a tsunami that follows an earthquake. that is indicative of the boom in stages of better moisture stages and perhaps even sturdy rains.

\section{Need for Disaster Management}

A catastrophe could be a flavoring or guy-made danger that causes severe within the functioning of a community or a society. Disaster brings with it splendid injury, loss, destruction and devastation to not most effective human lifestyles and assets however additionally to infrastructure, shipping networks and financial system. it's far important to allot the records and resources in a proper way such that the widest variety of the hundreds outlast the sick-effects of the catastrophe. even though it is not absolutely viable to keep away from the incidence of a catastrophe, the effect can honestly be minimized through creating proper techniques of focus of probable catastrophe and its consequences by using growing a system that channels this statistics to the involved government [6]. IoT will play an important role throughout this channeling and transmission of knowledge through the inexperienced use of existing technology [7].Catastrophe management is improbably significant to alive within the case of a catastrophe and can be described as the employer and control of assets and duties for handling with all humanitarian parts of emergencies specifically, awareness, reaction, and healing an honest thanks to reduce the impact of a sudden catastrophe.

\section{Problem Statement}

Design and Implementation of a system with the help of Raspberry-Pi that's interfaced with varied sensors (Temperature, Rain, Moisture, and Vibration). Real time info is to be collected by all the sensors and might be fetched by information superhighway Server.

This info is also accessed by the user through an internet browser, sending SMS alerts via an internet application by setting a selected triggering purpose for every device. 


\section{System Design}

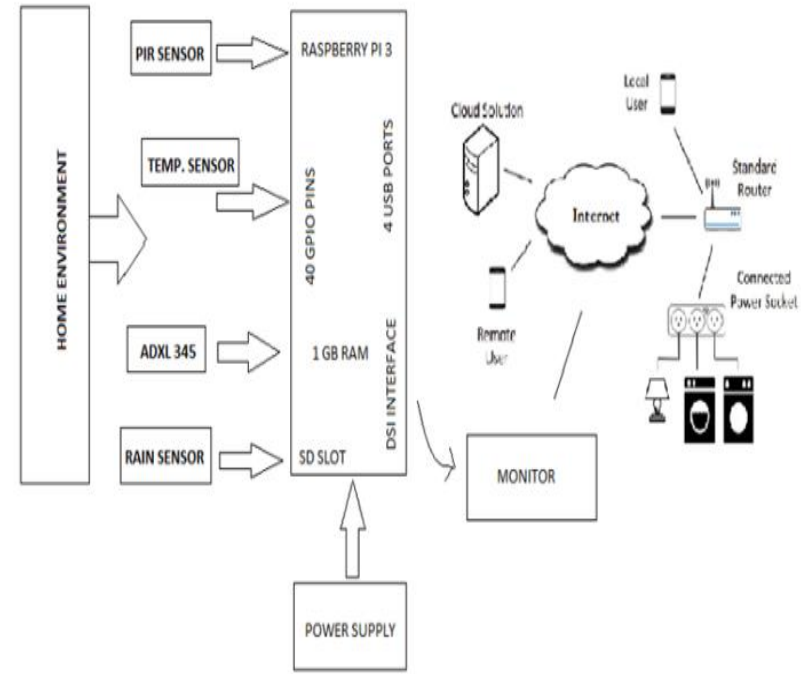

Fig. 1: System Architecture

\subsection{Hardware}

\subsubsection{Raspberry Pi}

It is an affordable, miniature card that connects to a computer or any other device used for display. It is an advanced and efficient tool that allows people to program in languages like Python and develop innovative tools. It can do anything from surfing the net and streaming high-definition videos, to growing spreadsheets and phrase-processing.

\subsubsection{Sensors}

\subsubsection{ADXL345 Digital Accelerometer Sensor}

The ADXL345 is a low-toned, three-axis MEMS accelerometer module with both I2C and SPI interfaces. The silicon wafer included within the sensor has a micro-machined shape. The structure is attached by poly-silicon springs which allow it to move smoothly in any direction when concerned to acceleration within the $\mathrm{X}, \mathrm{Y}$ or $\mathrm{Z}$ axis. This deviation triggers a modification in capacitance among constant plates connected to the lung structure. This alteration in capacitance on each axis is transformed to an output voltage proportional to the acceleration on that particular axis.

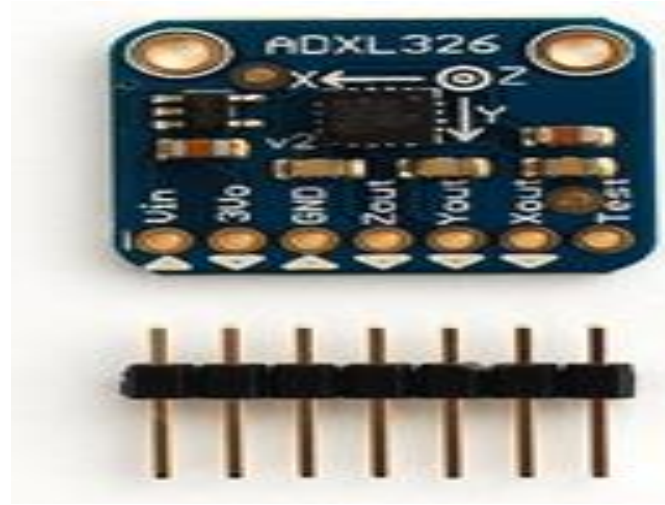

Fig. 2: Accelerometer Sensor

\subsubsection{PIR Motion Sensor}

Every object emits heat in the form of radiation when their temperatures are above the absolute zero. They actually work by detecting the energy given off with the aid of different gadgets. PIR sensors don't hit upon "warmness"; as an alternative, they discover the infrared radiation mediated by an object. The PIR sensors have various slots in it. Per slot is manufactured from a specific concrete that is perceptive to infrared radiation. While the sensor is vacant or inactive, per slot measures the equal amount of infrared radiation, which is the effective amount radiated from the room or partitions or exterior. When a warmish object like a mortal or an animal passes through it, it first pick off one half of the PIR sensor that results in a positive differential change between the two halves. When the object comes out of the sensing area, the opposite occurs, wherein the given sensor produces a negative differential alternate. These exchanged pulsations are then identified and realised.

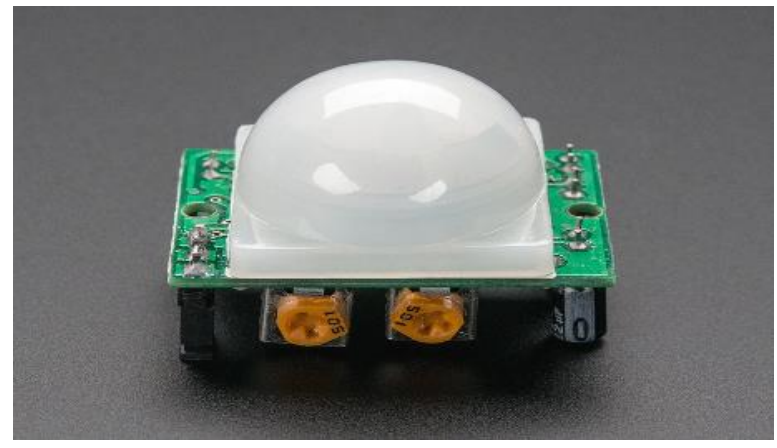

Fig. 3 : PIR Motion Sensor

\subsubsection{Rain Drop Sensor}

This module allows us to measure the weakness via analogue output pins and it produces a digital output while the lower limit of humidity is passed. The module is working on the LM393 OpAmp. It comprises of the electronics module and a imprinted control board that "accumulates" the drops of rain. As drops of rain are amassed on the board, they make tracks of parallel resistance which are then measured via the Op-Amp. The softer the resistance or more waterlog, the lower the voltage. Inversely, lessen the amount of waterlog, more the voltage at the analogue pin. An arid board, for instance, will drive the module to output $5 \mathrm{~V}$.

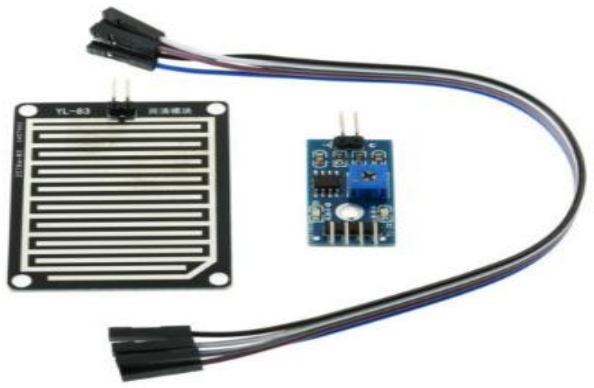

Fig. 4: Rain Drop Senso

\subsubsection{Temperature Sensor}

The major usage of DS18B20 is its direct-to-digital temperature device. This module has a unique 64-bit code hoarded in an onboard ROM. It can be powered on from $3 \mathrm{~V}$ to $5 \mathrm{~V}$ range. It can measure temperature from $-55^{\circ} \mathrm{C}$ to $+125^{\circ} \mathrm{C}$. Thermometer resolution is user-controlled from 9 to 12 bits. It converts temperature to 12-bit digital word in 750 milliseconds. It has useroperational non-volatile alarm settings. It has a good accuracy with $\pm 0.5^{\circ} \mathrm{C}$.Unalike analog temperature sensors, number crunching is performed by the sensor and the output is an actual temperature value. 

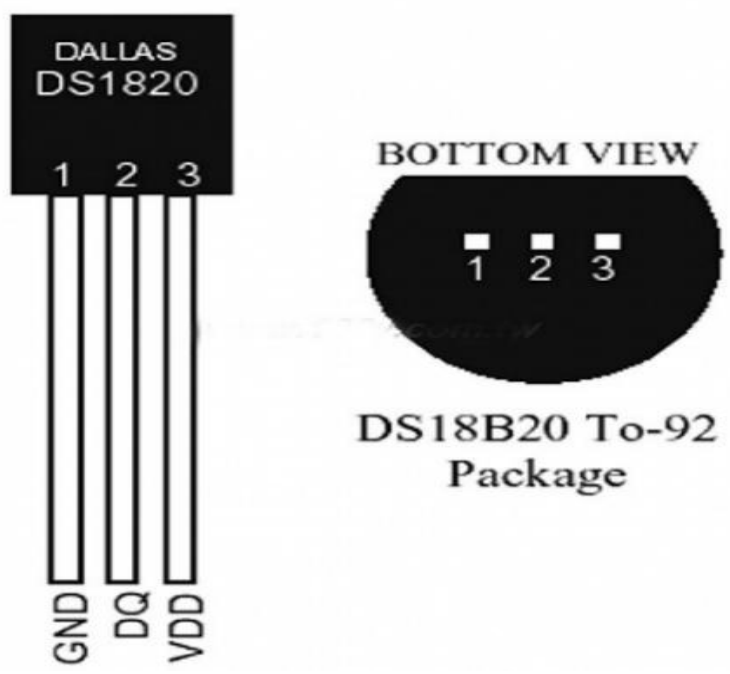

DS18B20 To-92 Package

Fig. 5: Temperature Sensor
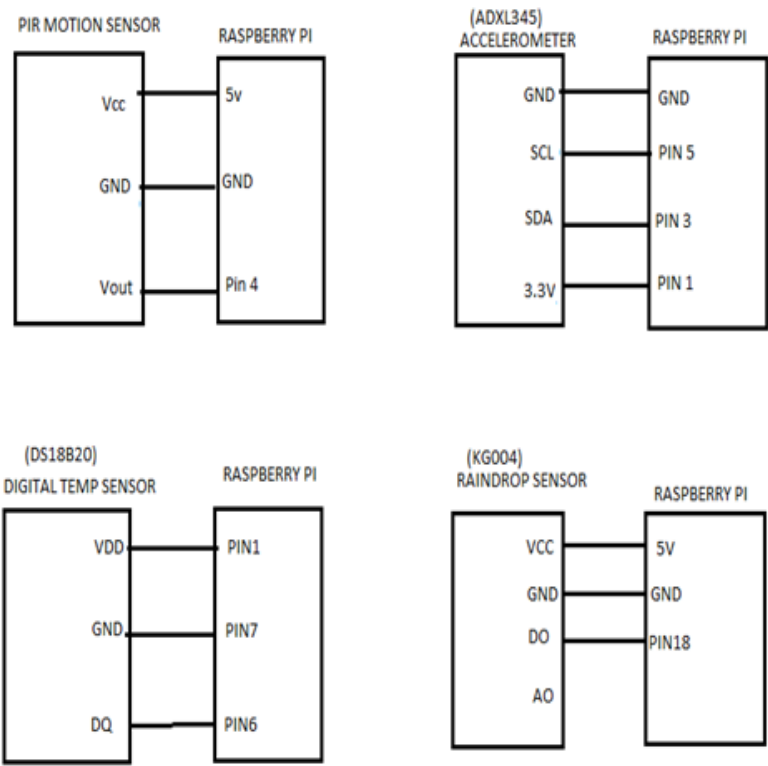

Fig. 6: Sensors Connections

\subsection{Technologies}

\subsubsection{Python}

Python is a predominantly used global purpose, high-level programing language. Its vogue ideology accentuates code readability, and its syntax allows programmers to precise ideas in lesser lines of code that might be attainable in languages such as C,C++,JavaScript or Java. The language delivers relevant constructs which are supposed to enable clearing programs on both a small and large scale. Python assists and upholds multiple programming paradigms, likewise as object-oriented, imperative and practical programming or proceeding styles. It has significant features of dynamic type system and involuntary memory management and comprising a giant and customary library

Program on how to read digital temperature detector values and send data to things speak platform and additionally to send an SMS alert using Twilio.

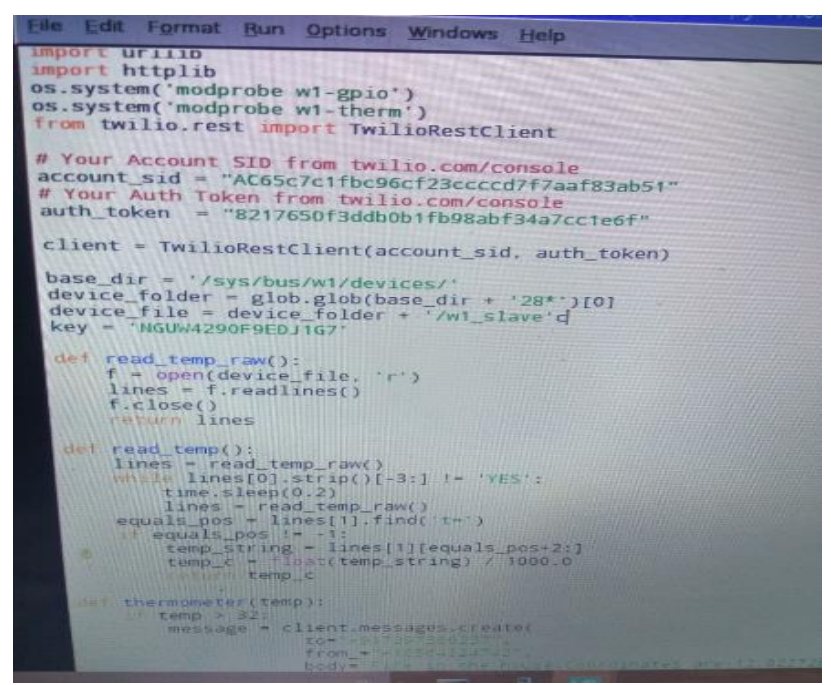

Fig. 7: Programming in Python

\subsubsection{TWILIO}

Twilio (Nexmo\&Tropo) provides APIs for Text Messaging, VoIP $\&$ Voice in the Cloud.

Sample Program on how to send SMS using Twilio.

For sending an SMS, make an HTTP POST request to the message resource.

https://api.twilio.com/201010401/Accounts/AC123456abc/Messages Open a file called send_sms.py and include the following lines:

\section{send_sms.py}

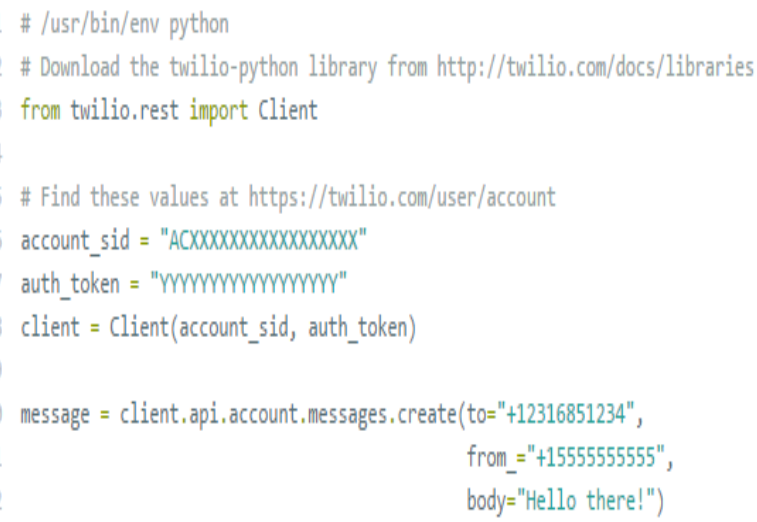

Fig. 8: Twilio Interface

A number should be a valid Twilio Phone number. The numeric digits can be any outgoing number. If you are exercising a Twilio Trial account like in this example, you can only send SMS messages to phone numbers that you have authenticated with Twilio. Phone numbers can be validated via your Twilio Account's Phone Numbers Page.

\subsubsection{ThingSpeak}

Open source data platform and API for the Internet of Things provides access to a broad kind of embedded devices and web services. ThingSpeak is associated in nursing open info platform and API for the IoT that allows you to assemble, store, analyze, visualize, and act on info from sensors or actuators, like Arduino, Raspberry $\mathrm{Pi}$, and different hardware. For example, with ThingSpeak you will be able to manufacture sensor logging apps, location-tracking apps, and a social network of things with regular status updates in such a way that you may have your home thermostat controlling itself based on your current location. 


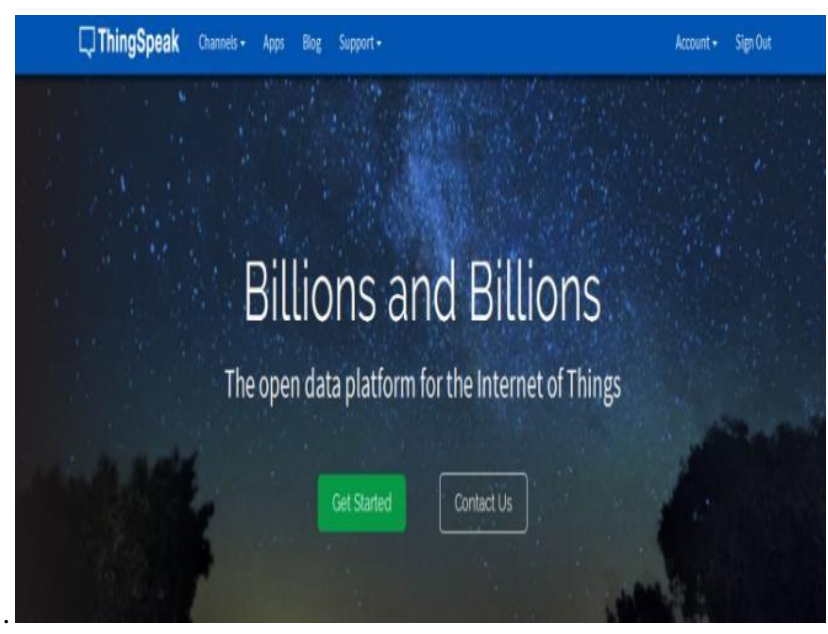

Fig. 10: ThingSpeak

\subsubsection{Channel Creation}

ThingSpeak channels stores the data sent from sensors and devices. We have created our channel as IoT

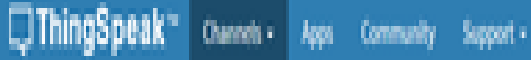

\section{My chantinels}

\section{Hrom}

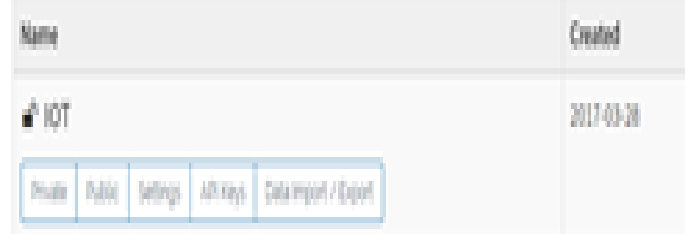

Fig. 11: Channel Creation

\subsubsection{API Keys}

API keys enable you to scratch info to a channel or read info from a private channel. API keys are self-generated when you made a fresh channel.

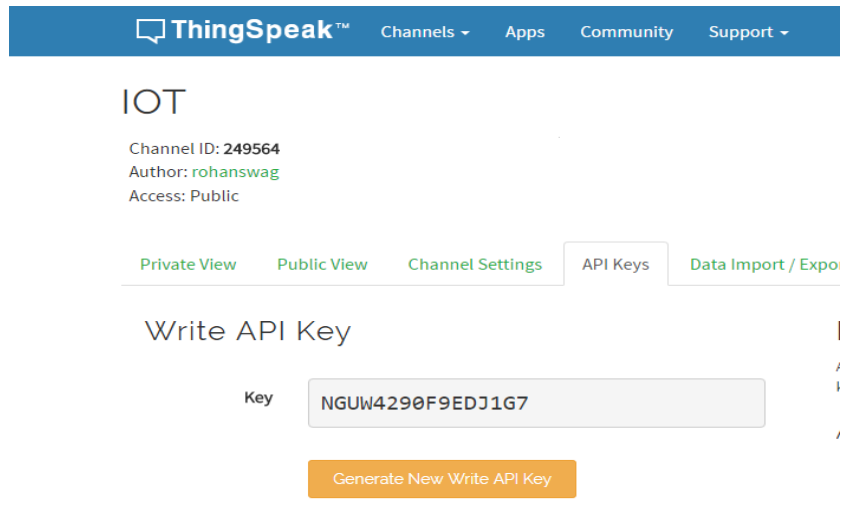

Read API Keys

\section{Key WG176311D5ZJOUOQ}

Fig. 12: API Keys

\section{Hardware And Software Specification}

For Raspberry-pi

1) Memory card of $8 \mathrm{~GB}$ or $4 \mathrm{~GB}$.

2) HDMI monitor for displaying.

3) Ethernet cable for providingweb access.

4) Keyboard and Mouse.

5) 5-Volt supply.

6) Sensors.

Software used:

Programming Language: Python

O.S: Raspbian

\section{User Interface}

\subsection{For Temperature Sensor}

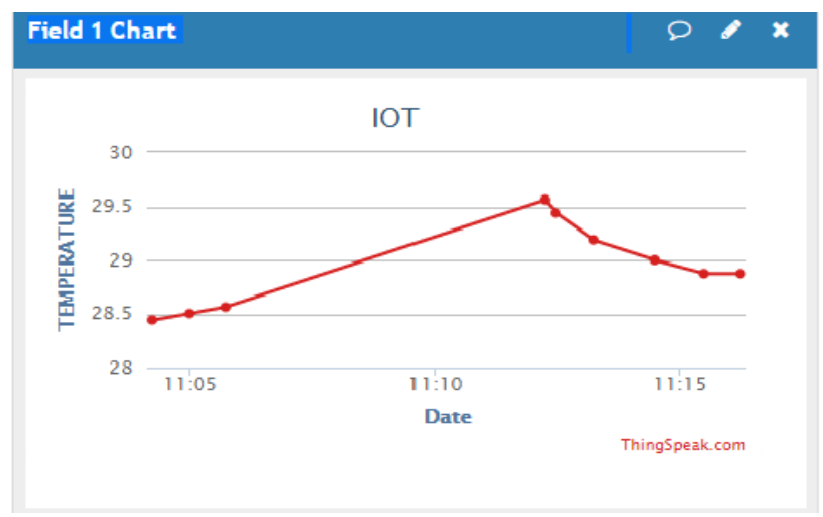

\subsection{For accelerometer}

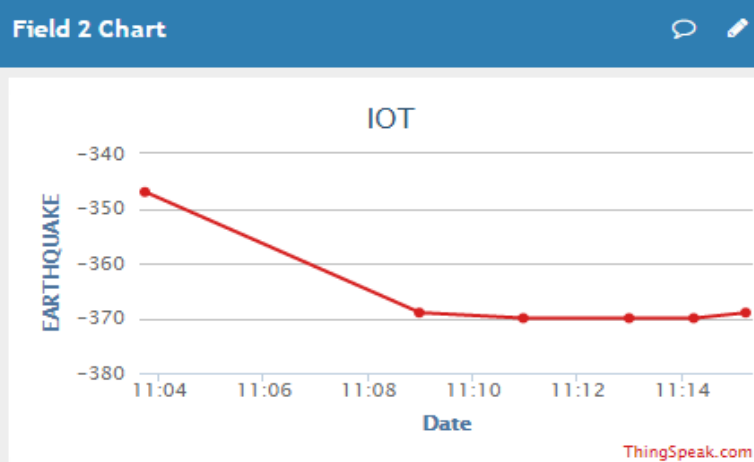

\subsection{For Motion Sensor}

Field 4 Chart

\section{IOT}

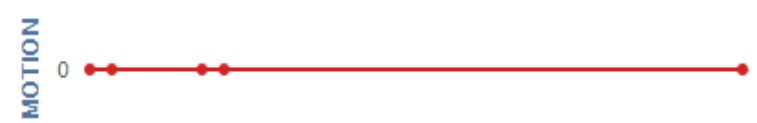

\begin{tabular}{|c|c|c|c|}
\hline & $11: 12$ & 11:14 & $11: 16$ \\
\hline
\end{tabular}




\subsection{For Rain Drop Sensor}

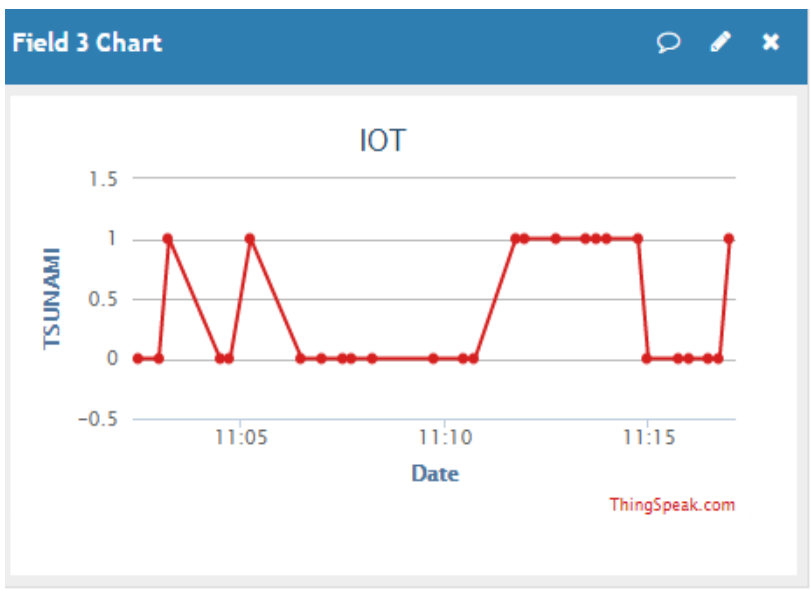

\subsection{Channel Location}

\section{Channel Location}

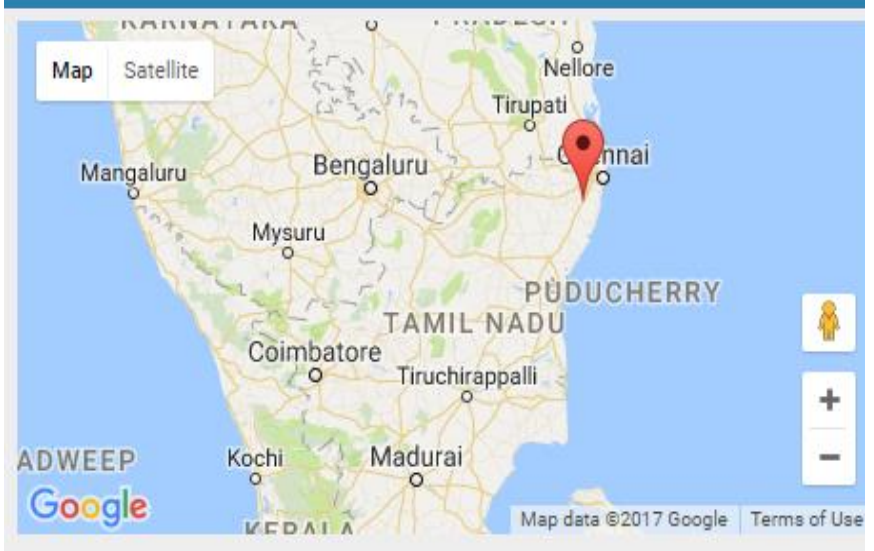

\section{Conclusion}

IoT has got off an upsurge of the knowledge business and remains in its preliminary stage. An occasional value module employed in IoT permitted systems has been designed and also constructed for the utilization for Disaster Management Team to rescue or safe the victims suffering in catastrophic events. Initial testing showed positive results. Coming work includes improvement of the network, dynamic adaption of modules to dynamic conditions and how to develop devoted protocol for disaster management.

\section{References}

[1] Federal Emergency Management Agency FEMA, US Department of Homeland Security, USA, 2001.

[2] J. Gubbi and R. Buyya, "Internet of things (IoT): A vision, architectural elements, and future directions," Future Generation Computer Systems 29, pp. 1645-1660, 2013.

[3] T.-Y. Chen, H.-W. Wei, N.-I. Hsu, and W.-K. Shih, "An IoT application of safe building in the ipv6 network environment," IEEE 37th Annual Computer Software and Applications Conference, pp. 748-753, 2013.

[4] A. Monzon, "Smart cities concept and challenges bases for the assessment of smart city projects," IEEE Smart Cities and Green ICT Systems (SMARTGREENS), pp. 1-11, May 2015.

[5] Zanella, N. Bui, A. Castellani, L. Vangelista, and M. Zorzi, "Internet of things for smart cities," Internet of Things Journal, IEEE, pp. 22-32, Feb. 2014

[6] Yusoff, I. S. Mustafa, S. Yussof, and N. M. Din, "Green cloud platform for flood early detection warning system in the smart city," Information Technology: Towards New Smart World (NSW NSW), IEEE, pp. 1-6, Feb. 2015.
[7] A. Zelenkauskaite, N. Bessis, S. Sotiriadis, and E Asimakopoulou, "Interconnectedness of complex systems of the internet of things through social network analysis for disaster management," Intelligent Networking and Collaborative Systems (INCoS), IEEE, pp. 503-508, 2012.

[8] T. Padmapriya, V.Saminadan, "Performance Improvement in long term Evolution-advanced network using multiple imput multiple output technique", Journal of Advanced Research in Dynamical and Control Systems, Vol. 9, Sp-6, pp: 990-1010, 2017.

[9] S.V.Manikanthan and V.Rama"Optimal Performance of Key Predistribution Protocol In Wireless Sensor Networks" International Innovative Research Journal of Engineering and Technology ,ISSN NO: 2456-1983,Vol-2,Issue -Special -March 2017 